\title{
Curbing the menace of building collapse in Nigeria
}

\author{
Michael O. Ajufoh ${ }^{1}$, W. A Gumau', Yaktor Joshua Inusa ${ }^{2}$ \\ ${ }^{1}$ Department of Architecture, Federal Polytechnic, Bauchi, Bauchi State, Nigeria \\ ${ }^{2}$ Department of Architecture, University of Jos, Jos, Plateau State, Nigeria
}

\begin{abstract}
Marcus Vitruvious polio, the Roman writer, Architect and Engineer, was famous for asserting in his book De Architectura that a structure must exhibit the three qualities of firmitas, utilitas and venustas - that is, it must be strong or durable, useful and beautiful. Hugo Alvar Aalto the Finnish Architect and designer, argued that the responsible designer/Architect must "do no harm" on the end users of such a building nor provide environments unsuitable for their use. Part of the federal Government of Nigeria's transformation agenda is the provision of quality and affordable infrastructure through Housing and Urban Development. But the spate of collapsed buildings in the country has reached an alarming rate. The incessant cases are enough reasons to declare a state of emergency in the construction industry. A building collapse could be a total or partial failure of one or more components of a building leading to the inability of the building to perform its principle function of safety and stability. In an attempt to find a solution to this menace, this paper therefore tries to examine some of the major causes of building collapse and tries to proffer remedial measures that may curb its devastating effects. The paper also briefly outlines its implications on real estate investment and also briefly tries to advice on procedures for rescuing people from collapsed buildings.
\end{abstract}

Keywords: real estate investment; urban development; Marcus Vitruvious; Hugo Alvar Aalto

\section{Preamble}

"The Federal Government has said that the success of its transformation agenda depends on infrastructure development. It added that though it has three other priorities in the next five years, including good governance, human capital and real sector development, premium is placed on infrastructure projects funding to impact positively on the realisation of the other priorities" (Chuka Uroko, Bussinesday, Friday, 15 March 2013).

\section{INTRODUCTION}

The pursuit of shelter for all mankind and his activities has always been a paramount issue. Buildings are constructed to serve as shelter for man, his properties and other activities therefore they must be properly planned, designed and erected to obtain desired satisfaction from the environment, (Ayuba, 2007; Olagunju, 2007; Akande, 2007).

However, recent events in places like Abuja, Lagos, Port Harcourt, Enugu, Aba, and other places in the country have seen these buildings as a growing cause of death, loss of property, and has left many people injured, (Ayuba, 2007).

In just 2005, more than 10 buildings collapsed in Lagos and Port Harcourt alone, not to mention those in Abuja, Kaduna and other places not publicized, (Agba, 2005; Awojobi, 
2005; Salau, 2005; Sanni, 2005). These buildings either collapsed at construction stage or after habitation (during the building life span), but studies have shown that some buildings in Nigeria collapses during construction stage, (Sanni 2005).

However, it is very important to note that buildings collapse due to a variety of reasons such as bad design, faulty construction, foundation failures, and extra ordinary loads, (Calvert, 2007; Onyemachi, et al, 2005). Earthquakes, hurricanes, tornadoes, floods, avalanches and other weather phenomenon have shown a far greater capacity for destruction, than society has for building structures that will withstand the weather or earth disruptions, (Haruna, 2007). So inasmuch as weather has a role to play as a natural cause for collapsed structures, the September $11^{\text {th }}$ shocker on the twin towers in New York City can be termed as a man-made destructive force. In ensuring qualitative housing and urban development, the federal government will do well by ensuring that the menace of collapsed buildings is completely dealt with, or it will simply be providing death traps for its citizens.

\section{CASE STUDIES FROM AROUND THE COUNTRY}

Building collapse is not peculiar to a state or region alone it's a common problem encountered all over the world. In Nigeria it is widespread in all major cities. It is, however, predominant in the south west and Lagos in particular.

"Lagos is worst hit. Barely seven months into the year, not less than 15 cases of building collapse had been recorded with about 30 lives lost and properties worth billions of naira lost. Scores are also rendered homeless each time a case of a collapsed building is recorded. The recent incident recorded in Lagos, penultimate Sunday, coming barely two weeks after a similar occurrence claimed eight lives in Ebutte Metta area of the state, was perhaps one collapse too many" (Daily Independent, Tuesday $5^{\text {th }}$ of November 2013).

Cases of building collapse are not restricted by climatology or level of urbanization as they cut across cultural and ethnical barriers. Many cases of building collapse have been reported in Nigeria. For instance, Folagbade (2001) and Chinwokwo (2000) enumerated forty-two (42) cases of building collapse as occurring between 1980 and 1999 in Nigeria while Makinde ( 2007) listed fifty-four (54) cases occurring between January 2000 and June 2007 alone. Building collapse has also been observed to cut across the different categories of building - private, corporate or public. Folagbade (2001) showed that of the twenty-five (25) reported cases of building collapse between 1980 and 1999 in Lagos State, private (76\%), corporate (12\%) and government or public buildings (12\%) accounted for these proportions. Also, building collapse is no respecter of size of the structure. Amusan (1991) reported that Barnawa flat disaster in 1977 was a three-storey building, a public building (Secondary School) which collapsed in March 1988 at Ibadan was two-storey building, and the collapsed show-room for cars in Lagos in 1987 was just a storey building while that of the Primary School in IIoabuchi, River State in July 1991 was a bungalow building. 
Table 1. Some of the Reported Cases of Collapsed Build dings in Nigeria (1974-2010).

\begin{tabular}{|c|c|c|c|c|c|}
\hline $\mathbf{S} / \mathbf{N}$ & $\begin{array}{l}\text { TYPE OF } \\
\text { BUILDING } \\
\text { STRUCTURE }\end{array}$ & $\begin{array}{ll}\text { LOCATION } & \text { OF } \\
\text { BUILDING } & \end{array}$ & $\begin{array}{l}\text { DATE OF } \\
\text { COLLAPSE }\end{array}$ & $\begin{array}{l}\text { SUSPECTED } \\
\text { CAUSE(S) }\end{array}$ & $\begin{array}{l}\text { REMARKS } \\
\text { (LIVES } \\
\text { LOST) }\end{array}$ \\
\hline 1. & $\begin{array}{l}\text { Multi-storey } \\
\text { Building under } \\
\text { construction }\end{array}$ & $\begin{array}{l}\text { Mokola, Ibadan, Oyo } \\
\text { State }\end{array}$ & October 1974 & $\begin{array}{l}\text { Excessive } \\
\text { loading/structural failure }\end{array}$ & 27 people \\
\hline 2. & $\begin{array}{l}\text { Partial collapse } \\
\text { of an Hostel } \\
\text { Building }\end{array}$ & $\begin{array}{l}\text { O.A.U., } \\
\text { Ile-ife }\end{array}$ & $\begin{array}{l}1976 \\
(1975 / 76 \\
\text { Academic } \\
\text { Session }\end{array}$ & Structural Failure & Nil \\
\hline 3. & $\begin{array}{l}\text { Residential } \\
\text { Building }\end{array}$ & $\begin{array}{l}\text { Barnawa housing Estate, } \\
\text { Kaduna, State }\end{array}$ & August 1977 & Faulty design & 28 people \\
\hline 4. & School Building & $\begin{array}{l}\text { Government Secondar } \\
\text { school, makarfi, Kaduna } \\
\text { State }\end{array}$ & July 1977 & Carelessness & 7 people \\
\hline 5. & $\begin{array}{l}\text { 3-Storey } \\
\text { Residential } \\
\text { Building }\end{array}$ & $\begin{array}{l}\text { Barnawa Housing Estate } \\
\text { Kaduna }\end{array}$ & 1980 & Faulty Structural design & 6 people \\
\hline 6. & $\begin{array}{l}\text { Storey } \\
\text { Residential } \\
\text { Building }\end{array}$ & $\begin{array}{l}\text { Allen Avenue, Ikeja } \\
\text { Lagos }\end{array}$ & Jnauary 1985 & Excessive Loading & Nil \\
\hline 7. & $\begin{array}{l}\text { Residential } \\
\text { Building }\end{array}$ & Adeniji Adele, Lagos & $\begin{array}{l}\text { February } \\
1985\end{array}$ & Excessive Loading & 2 people \\
\hline 8. & $\begin{array}{l}\text { Residential } \\
\text { Building }\end{array}$ & Ojuelegba Area, Lagos & May 18,1985 & Rainstorm & Nil \\
\hline 9. & $\begin{array}{l}\text { Residential } \\
\text { Building } \\
\text { (Uncompleted } 4 \\
\text { Storey Building) } \\
\end{array}$ & Iponri, Lagos & May 20,1985 & Structural Failure & 13 people \\
\hline 10. & $\begin{array}{l}\text { Residential } \\
\text { Building }\end{array}$ & Victoria Island, Lagos & July 18,1985 & Excessive Loading & $\begin{array}{l}13 \text { people } \\
\text { (all of the } \\
\text { same family }\end{array}$ \\
\hline 11. & $\begin{array}{l}\text { Residential } \\
\text { Building }\end{array}$ & Gboko, Benue State & $\begin{array}{l}\text { September, } \\
1985\end{array}$ & Carelessness & 1 person \\
\hline 12. & $\begin{array}{l}\text { Residential } \\
\text { Building }\end{array}$ & Allen Avenue & 1985 & Carelessness & Nil \\
\hline 13. & $\begin{array}{l}\text { Residential } \\
\text { Building }\end{array}$ & Adeniji Adele, Lagos & 1985 & $\begin{array}{l}\text { Faulty design/ } \\
\text { Carelessness }\end{array}$ & 2 people \\
\hline
\end{tabular}




\begin{tabular}{|c|c|c|c|c|c|}
\hline 14. & $\begin{array}{ll}\text { High } & \text { Court } \\
\text { Building } & \end{array}$ & Isala Area, Imo State & $\begin{array}{l}\text { February } \\
1986\end{array}$ & Structural Failure & 2 people \\
\hline 15. & $\begin{array}{l}\text { Mosque } \\
\text { Building }\end{array}$ & Oshogbo, Osun State & May, 1986 & Structural Failure & 2 people \\
\hline 16. & $\begin{array}{l}\text { Residential } \\
\text { Building }\end{array}$ & $\begin{array}{l}\text { Ona Street } \\
\text { Enugu State }\end{array}$ & 1986 & No Investigation & 2 people \\
\hline 17. & $\begin{array}{l}\text { 2-Storey } \\
\text { Building under } \\
\text { construction }\end{array}$ & Agege, Lagos & May 9, 1987 & Structural Fault & 2 people \\
\hline 18. & $\begin{array}{l}\text { Residential } \\
\text { Building }\end{array}$ & $\begin{array}{l}\text { Idusagbe Lane, Idumota } \\
\text { Lagos }\end{array}$ & $\begin{array}{l}\text { September } \\
14,1987\end{array}$ & No Structural Design & 17 people \\
\hline 19. & $\begin{array}{l}\text { Commercial } \\
\text { Building }\end{array}$ & Ikorodu Road, Lagos & $\begin{array}{l}\text { September } \\
1987\end{array}$ & Rainstorm & 4 people \\
\hline 20. & $\begin{array}{l}\text { Residential } \\
\text { Building }\end{array}$ & $\begin{array}{l}\text { Calabar, Cross } \\
\text { State }\end{array}$ & $\begin{array}{l}\text { October } 9, \\
1987\end{array}$ & Rainstorm & 3 people \\
\hline 21. & \begin{tabular}{ll|} 
6-storey & Hotel \\
Building &
\end{tabular} & $\begin{array}{l}\text { Akinwunmi street, } \\
\text { Mende Village, Lagos }\end{array}$ & October 1989 & Faulty design & Nil \\
\hline 22. & $\begin{array}{l}\text { Bungalow } \\
\text { School Building }\end{array}$ & $\begin{array}{ll}\text { Port Harcourt, Rivers } \\
\text { State }\end{array}$ & June 15,1990 & $\begin{array}{l}\text { Ignorance of the owner } \\
\text { and absence of structural } \\
\text { design }\end{array}$ & Nil \\
\hline 23. & $\begin{array}{ll}\text { 6-storey } & \text { Hotel } \\
\text { Complex } & \end{array}$ & $\begin{array}{l}\text { Okupe Estate Maryland, } \\
\text { Lagos }\end{array}$ & 1993 & Structural Failure & Not Known \\
\hline 24. & $\begin{array}{l}\text { Multi-purpose } \\
\text { Indoor Sports } \\
\text { Complex Storey }\end{array}$ & Area 10, Abuja & March 1993 & $\begin{array}{l}\text { Structural failure/Poor } \\
\text { workmanship }\end{array}$ & Not reported \\
\hline 25. & $\begin{array}{l}\text { Multi-storey } \\
\text { Building } \\
\text { NICON-NOGA } \\
\text { Staff Housing } \\
\text { Project }\end{array}$ & Karo, Abuja & $\begin{array}{l}\text { March } 25, \\
1993\end{array}$ & $\begin{array}{l}\text { Structural failure/use of } \\
\text { incompetent people for } \\
\text { Supervision }\end{array}$ & Not reported \\
\hline 26. & $\begin{array}{l}\text { A Mosque } \\
\text { Building under } \\
\text { construction }\end{array}$ & Abeokuta Ogun State & 1995 & $\begin{array}{l}\text { Structural failure/use of } \\
\text { incompetent people for } \\
\text { Supervision }\end{array}$ & 2 people \\
\hline 27. & $\begin{array}{l}\text { Storey Building } \\
\text { (under } \\
\text { construction) }\end{array}$ & Central Lagos & $\begin{array}{l}\text { October } 5, \\
1995\end{array}$ & $\begin{array}{l}\text { Poor } \quad \text { workmanship/ } \\
\text { structural failure }\end{array}$ & 10 people \\
\hline 28. & $\begin{array}{l}\text { 3-storey Church } \\
\text { Building }\end{array}$ & Lagos & $\begin{array}{l}\text { October } 30, \\
1995\end{array}$ & Structural failure & 6 people \\
\hline 29. & School Building & $\begin{array}{l}\text { Alagbado Area, Ibadan } \\
\text { Oyo State }\end{array}$ & October 1995 & Poor workmanship & Nil \\
\hline 30. & $\begin{array}{l}\text { 3-Storey } \\
\text { Building }\end{array}$ & $\begin{array}{l}\text { Oke Igbala Area, Ibadan } \\
\text { Oyo State }\end{array}$ & October 1995 & Structural failure & 6 people \\
\hline 31. & $\begin{array}{l}\text { 1-stroey } \\
\text { Building under } \\
\text { construction but } \\
\text { being used as } \\
\text { church (Earlier } \\
\text { approved as a } \\
\text { bungalow }\end{array}$ & $\begin{array}{lr}\text { Olowookere } & \text { street, } \\
\text { Mafoluku, } & \text { Oshodi, } \\
\text { Lagos }\end{array}$ & June 1996 & Structural Weakness & 7 people \\
\hline 32. & $\begin{array}{l}\text { Storey Building } \\
\text { under } \\
\text { construction }\end{array}$ & Lagos State & $\begin{array}{l}\text { March } \\
1996\end{array}$ & Structural failure & $\begin{array}{l}\text { People only } \\
\text { injured }\end{array}$ \\
\hline
\end{tabular}




\begin{tabular}{|c|c|c|c|c|c|}
\hline 33. & $\begin{array}{l}\text { 6-Storey } \\
\text { Building under } \\
\text { construction } \\
\text { (being used as } \\
\text { Nursery/Primary } \\
\text { School) }\end{array}$ & Lagos State & $\begin{array}{l}\text { October, } \\
1996\end{array}$ & $\begin{array}{l}\text { Use of quacks/Structural } \\
\text { Failure }\end{array}$ & 1 person \\
\hline 34. & $\begin{array}{l}\text { 2-Storey } \\
\text { Building }\end{array}$ & $\begin{array}{l}\text { Amu Street, Mushin, } \\
\text { Lagos }\end{array}$ & June, 1997 & $\begin{array}{l}\text { Use of poor } \\
\text { materials/structural } \\
\text { failure }\end{array}$ & Nil \\
\hline 35. & Duplex Building & $\begin{array}{l}\text { Gwarinpa Area, FCT, } \\
\text { Abuja }\end{array}$ & 1998 & Structural failure & 2 people \\
\hline 36 & $\begin{array}{l}\text { 3-Storey } \\
\text { Residential } \\
\text { Building }\end{array}$ & Ibadan, Oyo State & 1998 & $\begin{array}{l}\text { Faulty, Design/Poor } \\
\text { workmanship }\end{array}$ & $\begin{array}{l}\text { Several } \\
\text { people }\end{array}$ \\
\hline 37. & $\begin{array}{l}\text { 4-Storey Church } \\
\text { Building (under } \\
\text { construction) }\end{array}$ & Akure, Ondo State & $\begin{array}{l}\text { October } 1, \\
1998\end{array}$ & $\begin{array}{ll}\text { Structural } & \text { failure/Poor } \\
\text { Supervision } & \end{array}$ & 8 People \\
\hline 38. & $\begin{array}{l}\text { 2-Storey } \\
\text { Residential } \\
\text { Building }\end{array}$ & $\begin{array}{l}\text { Rd 3, Plot 10, Funbi } \\
\text { Fagun Street, Abeokuta, } \\
\text { Ogun State }\end{array}$ & $\begin{array}{l}\text { November, } \\
1998\end{array}$ & $\begin{array}{l}\text { Use of } \quad \text { poor } \\
\text { materials/structural } \\
\text { failure }\end{array}$ & Nil \\
\hline 39. & $\begin{array}{l}\text { 3-Storey } \\
\text { Residential } \\
\text { Building }\end{array}$ & $\begin{array}{l}\text { Ojuelegba, Western } \\
\text { Avenue, Surulere, Lagos } \\
\text { State }\end{array}$ & 1999 & $\begin{array}{l}\text { Carelessness/use of poor } \\
\text { materials }\end{array}$ & 4 people \\
\hline 40. & $\begin{array}{l}\text { 1-Storey } \\
\text { Residential }\end{array}$ & $\begin{array}{l}\text { Adeola Odeku Street, } \\
\text { Victoria Island, Lagos }\end{array}$ & 1999 & Rainstom & Not reported \\
\hline 41. & $\begin{array}{l}\text { 3-Storey } \\
\text { Residential } \\
\text { Building }\end{array}$ & $\begin{array}{l}\text { Charity Road, Oko-Oba, } \\
\text { Lagos }\end{array}$ & June 1999 & Structural failure & Nil \\
\hline 42 & $\begin{array}{l}\text { 3-Storey } \\
\text { Residential }\end{array}$ & $\begin{array}{ll}\text { Four-square } & \text { Gospel } \\
\text { Church Abuja } & \end{array}$ & $\begin{array}{l}\text { October, } \\
1999\end{array}$ & $\begin{array}{l}\text { Faulty design/ } \\
\text { Implementation }\end{array}$ & $\begin{array}{l}\text { Not } \\
\text { Available }\end{array}$ \\
\hline 43. & $\begin{array}{l}\text { 1-Storey } \\
\text { Residential } \\
\text { Building }\end{array}$ & $\begin{array}{l}\text { Obawole street, Iju, } \\
\text { Agege, Lagos }\end{array}$ & $\begin{array}{l}\text { October, } \\
1999\end{array}$ & Structural failure & Nil \\
\hline 44. & $\begin{array}{l}\text { 3-Storey } \\
\text { Residential } \\
\text { Building }\end{array}$ & Iju-Isaga, Lagos & August, 1999 & $\begin{array}{l}\text { Structural } \\
\text { Faulty/Rainstorm }\end{array}$ & 35 people \\
\hline 45. & $\begin{array}{l}\text { 2-Storey } \\
\text { Residential } \\
\text { Building }\end{array}$ & $\begin{array}{l}\text { Dawodu street, Ifo, } \\
\text { Ogun State }\end{array}$ & $\begin{array}{l}\text { October, } \\
1999\end{array}$ & Rainstorm & 20 people \\
\hline 46. & $\begin{array}{l}\text { Residential } \\
\text { Storey Building }\end{array}$ & Idi-Oro, Mushin, Lagos & 2000 & $\begin{array}{l}\text { Faulty } \\
\text { Design/Carelessness }\end{array}$ & $\begin{array}{l}\text { Not } \\
\text { Available }\end{array}$ \\
\hline 47. & $\begin{array}{l}\text { Estate } \\
\text { Building }\end{array}$ & $\begin{array}{l}\text { Ajah, Along Lekki } \\
\text { Road, Lagos }\end{array}$ & April, 2000 & Structural Failure & Nil \\
\hline 48. & $\begin{array}{l}\text { 2-Storey } \\
\text { Mosque } \\
\text { Building }\end{array}$ & $\begin{array}{l}21, \quad \text { Buhari } \quad \text { Street } \\
\text { Mushin, Lagos }\end{array}$ & April 2001 & $\begin{array}{l}\text { Unauthorized Conversion } \\
\text { of former Bungalow to 2- } \\
\text { Storey Building }\end{array}$ & 7 people \\
\hline 49. & $\begin{array}{l}\text { 1-Storey } \\
\text { Residential } \\
\text { Building (under } \\
\text { construction) }\end{array}$ & Iwoye-Ijesa, Osun State & 2001 & $\begin{array}{l}\text { Structural failure/ use of } \\
\text { quacks for supervision }\end{array}$ & 7 people \\
\hline 50. & $\begin{array}{l}\text { Multi-storey } \\
\text { Commercial } \\
\text { Residential } \\
\text { Building }\end{array}$ & Ebute-Meta, Lagos & 2007 & $\begin{array}{l}\text { Unauthorized } \\
\text { conversion/poor } \\
\text { supervision/use of quality } \\
\text { materials }\end{array}$ & $\begin{array}{l}\text { Several } \\
\text { people }\end{array}$ \\
\hline
\end{tabular}




\begin{tabular}{|c|c|c|c|c|c|}
\hline 51. & $\begin{array}{l}\text { Multi-Storey } \\
\text { Building }\end{array}$ & Kano & 2007 & $\begin{array}{l}\text { Faulty design/structural } \\
\text { failure }\end{array}$ & $\begin{array}{l}\text { Several } \\
\text { people }\end{array}$ \\
\hline 52. & $\begin{array}{l}\text { A Building } \\
\text { being used as } \\
\text { Nursery/Primary } \\
\text { School }\end{array}$ & $\begin{array}{l}\text { Olomi Area Ibadan, Oyo } \\
\text { State }\end{array}$ & $\begin{array}{l}\text { March 25, } \\
2008\end{array}$ & $\begin{array}{l}\text { Use of poor materials } \\
\text { carelessness }\end{array}$ & 13 pupils \\
\hline 53. & $\begin{array}{l}\text { 5-Storey } \\
\text { Shopping } \\
\text { Complex } \\
\text { Building under } \\
\text { construction }\end{array}$ & Wuse Area, Abuja & $\begin{array}{l}2^{\text {nd }} \text { August, } \\
2008\end{array}$ & $\begin{array}{l}\text { Structural failure } \\
\text { incompetency/bad } \\
\text { workmanship }\end{array}$ & $\begin{array}{l}2 \text { people } \\
\text { injured and } \\
100 \text { people } \\
\text { trapped. }\end{array}$ \\
\hline 54. & $\begin{array}{l}\text { 2-Storey } \\
\text { Residential } \\
\text { Building under } \\
\text { construction }\end{array}$ & $\begin{array}{l}\text { Asero Area, Abeokuta } \\
\text { Ogun State }\end{array}$ & $\begin{array}{rr}30^{\text {th }} & \text { August } \\
2008 & \end{array}$ & $\begin{array}{l}\text { Contravening the given } \\
\text { planning Approval, use } \\
\text { of substandard materials } \\
\text { incompetency, etc. }\end{array}$ & 2 people \\
\hline 55. & $\begin{array}{l}\text { 6-Storey } \\
\text { LAUTECH } \\
\text { Teaching } \\
\text { Hospital } \\
\text { Complex under } \\
\text { construction }\end{array}$ & Ogbomoso, Oyo State & $\begin{array}{l}19^{\text {th }} \text { February, } \\
2009\end{array}$ & $\begin{array}{lr}\text { Use of } & \text { substandard } \\
\text { materials, } & \text { poor } \\
\text { workmanship/supervision }\end{array}$ & 5 people \\
\hline 56 & A wall fence & $\begin{array}{l}\text { Aghaji crescent, GRA, } \\
\text { Enugu }\end{array}$ & $\begin{array}{l}10^{\text {th }} \text { August, } \\
2009\end{array}$ & No proper drainage & 1 person \\
\hline 57 & $\begin{array}{l}\text { Uncompleted } \\
\text { Building }\end{array}$ & $\begin{array}{l}\text { Oke Padre Street, } \\
\text { Ita-morin, Abeokuta }\end{array}$ & $\begin{array}{l}18^{\text {th }} \text { October, } \\
2009\end{array}$ & $\begin{array}{lr}\text { Use of } & \text { substandard } \\
\text { materials, } & \text { hasty } \\
\text { construction } & \end{array}$ & $\begin{array}{l}3 \text { people, } 11 \\
\text { injured }\end{array}$ \\
\hline 58 & $\begin{array}{l}\text { Building under } \\
\text { Construction }\end{array}$ & $\begin{array}{l}\text { Isopakodowo Street } \\
\text { Cairo, Oshodi, Lagos }\end{array}$ & $\begin{array}{l}26^{\text {th }} \quad \text { April } \\
2010\end{array}$ & $\begin{array}{l}\text { Use of Substandard } \\
\text { building materials }\end{array}$ & $\begin{array}{l}4 \text { people, } 12 \\
\text { injured }\end{array}$ \\
\hline 59 & $\begin{array}{l}\text { Uncompleted } \\
\text { Storey building }\end{array}$ & $\begin{array}{l}\text { Adenike Street off New } \\
\text { market, Oniru Estate, VI }\end{array}$ & June, & $\begin{array}{l}\text { Use of } \begin{array}{r}\text { Substandard } \\
\text { building materials, }\end{array} \\
\text { Non-compliance } \\
\text { house-owners a } \\
\text { developers with approved } \\
\text { building plan and weak } \\
\text { structure }\end{array}$ & $\begin{array}{l}1 \text { person, } 2 \\
\text { injured }\end{array}$ \\
\hline 60 & $\begin{array}{l}\text { Uncompleted } \\
\text { 4-Storey } \\
\text { Building }\end{array}$ & $\begin{array}{l}2 \text { Okolie Street, off } \\
\text { Gimbiya Street in Abuja. }\end{array}$ & $\begin{array}{l}11^{\text {th }} \text { August, } \\
2010\end{array}$ & $\begin{array}{l}\text { Substandard materials } \\
\text { and disregard for } \\
\text { building regulations }\end{array}$ & $\begin{array}{l}23 \text { people, } 11 \\
\text { injured }\end{array}$ \\
\hline 61 & $\begin{array}{l}4 \\
\text { Building }\end{array}$ & $\begin{array}{l}24 \text { Alli Street off Tinubu } \\
\text { Street, V. I. }\end{array}$ & $\begin{array}{l}28^{\text {th }} \\
\text { September, } \\
2010\end{array}$ & $\begin{array}{l}\text { Structural } \\
\text { Defects/overloading }\end{array}$ & 3 people \\
\hline
\end{tabular}

Source: F. I. Olabosipo and O. O. Adedamola, 2010.

Folagbade (2001) also reports that the Abuja building which collapsed in March, 1993 and the one at Ojuelegba in 1999 were both multi-storey buildings. The memory of the incidents of two separate building collapses that occurred at Ebute-Meta area of Lagos State and Kano State which killed several people in 2007 still lingers on. Also reported was the fence of a Nursery and Primary School that collapsed at Olomi area, Ibadan, in March, 2008, thereby killing thirteen (13) pupils of the School. The death of over 50 students of Saque Comprehensive College, Port Harcourt in 1990 was as a result of the owner attempting to construct additional floors on structurally unsafe walling. Similar trends of conversion were observed in a collapsed Mosque building in Mushin area, Lagos in 2001 and multi-storey commercial/residential building in Ebute-Meta also in Lagos state in which several people were killed. Some of the cases of building collapse are also as a result of ignorance on the 
part of developers and unauthorized conversion of buildings. Amusan (1991) asserts that the 1988 building collapse at Mushin, Lagos occurred when an attempt was made to raise the existing building by another floor. Also, operational conversion caused the collapsed school building at PortHarcourt. Fagbenle and Oluwunmi (2010).

Nigerian Building Collapses In 2011

\begin{tabular}{|c|c|c|c|c|c|c|}
\hline STATE & Date & Storeys & Location & Deaths & Injuries & Constuction Ongoing/Old \\
\hline Enugu & April/May 2011 & Up to 4 & Three (3) locations & - & & Ongoing \\
\hline Sokoto & $20 / 6 / 11$ & Mud wall & Sokoto Wall & 2 & & Old \\
\hline Abuja Outskirt & $29 / 6 / 11$ & 2 & Mararaba Bank & 2 & & Ongoing \\
\hline Abuja & $19 / 8 / 11$ & 3 & Mpape Hospital & 2 & & Ongoing \\
\hline Oyo & $2 / 7 / 11$ & & Ibadan & & & \\
\hline Anambra & $5 / 8 / 11$ & 2 & Awka Residential & & 17 & Ongoing \\
\hline \multirow[t]{10}{*}{ Lagos } & $13 / 3 / 11$ & 5 & Ikeja Hotel & & 1 & Ongoing \\
\hline & $5 / 7 / 11$ & 4 & Idumota Residential & 5 & & Old \\
\hline & $15 / 7 / 11$ & 2 & Ketu Residential & 2 & & Ongoing \\
\hline & $20 / 6 / 11$ & 3 & Island cold-room & & & New \\
\hline & $7 / 10 / 11$ & 3 & Ajao Estate & - & & Ongoing \\
\hline & $20 / 8 / 11$ & 3 & Lagos Isl & & 4 & \\
\hline & $26 / 8 / 11$ & 6 & Aderigbe str & - & & Old \\
\hline & $26 / 10 / 11$ & 6 & Maryland & - & & Old \\
\hline & $29 / 11 / 11$ & & Ikeja & - & & Old \\
\hline & $5 / 12 / 11$ & & Badagry School & - & 3 & Old \\
\hline
\end{tabular}

Source; Danladi Matawal, 2013.

\section{CAUSES OF BUILDING COLLAPSE}

In Nigeria building failures have been traced to the root causes which includes;

- Design faults - $50 \%$

- Faults on construction site $-40 \%$

- And product failure - $10 \%$

(Oyewande, 1982; Ayininuola and Olalusi, 2004)

Hall, (1984), agreed and equally ascribed faulty design, faulty execution of work and use of faulty materials to be major causes of building collapse. Causes can then generally be classified under six major factors;

1. Bad design: these include architectural designs and other professional inputs. Starting a design without proper feasibility studies, soil tests and analysis etc. Also, inadequate or poor design details, poor supervision, substandard materials specification and faulty or lack of schedules.it also includes errors of computations, use of inaccurate data etc.

2. Faulty construction: studies have revealed that faulty construction contributes up to $40 \%$ to structural failures in building collapse occurrences. These may arise due to failure of contractors to build according to the plans and specification or also due to 
poor mixing and placement of concrete.it may also include unstable bearing support for formwork or early removal.

3. Poor quality of materials and construction methods: the use of inferior materials probably has a $10 \%$ causative effect on the collapse of buildings (Oyewande 1992). Most block industries in Nigeria prefer profit to safety hence the precarious desire to mould as many blocks as possible from a single bag of cement.in some other cases you find out that most contractors prefer to use substandard steel for reinforcements.

4. Fire outbreak: today most of the available building materials in the developing countries are not only flammable but also encouraging the spread of fire (Adedoyin, 1983, Olagunju, 2002). This is the reason why a little fire ignition spreads very fast into large scale fire development in buildings. When the fire is blown out it weakens the structures reinforcements and concrete. It becomes even worse when the steel reinforcements are exposed directly to the fire, they may fail in the process to provide the necessary support for both the live and dead loads. In the event it may lead to partial or total collapse of the building. It makes it very important then to use materials with high fire resistant characteristics for building constructions. Also professionals should be conscious of this and apply the right specifications to them.

5. Natural phenomena: this includes the occurrence of floods, storm, exceptionally high wind or hurricane (Katrina, Ike, Gustav, Anna, Norbert, etc.), thunder, lightning, volcanic eruption and also earthquakes. These are factors that greatly affect building collapse, though most of them cannot be related to the Nigerian experience.

\section{REMEDIAL MEASURES}

a. Soil tests, Environmental impact assessment (EIA) and structural analysis needs to be made mandatory to be submitted along with the building plans to planning authorities by building approval seekers.

b. All plan for approval must be made to pass through all the registered professionals associated with the building industry before its final approval.

c. All plans for approval must be ensured to be in compliance with the new building code and all the affected local government's bye laws.

d. Inspection teams must be made to regularly inspect all construction works in their locality with the aim of enforcing the building code and local bye laws.

e. All professional bodies related to the construction industry should embark on enlightenment campaigns jointly for their members and also the public so as to create awareness on the evils and risks involved in the use of unqualified hands or better known as quacks.

\section{IMPLICATIONS ON REAL ETATE}

The implication of building collapse on real estate investments are numerous. The effects are felt in virtually all sectors of human endeavour in particular as it affects real estate investment are as follows;

1. Cost in real terms

2. Cost in relation to materials

3. Loss of human lives

4. Loss of capital invested in the property

5. Loss in rents return /profits etc. 
6. Loss of intrest on capital used for investing in the property;

7. Depreciation of value in the property

8. Loss of title to land.

\section{RESCUE FROM COLLAPSED BUILDINGS}

As many as one third of all building collapse victims, that are rescued, are found in spaces created by the way that building materials generally fall (Staten 2007; Mendis, 2007). Most of the collapse configurations that occur create voids in which people may be trapped and remain alive.

Most rescue experts agree that building collapse extrication must be a process of vertical removal, rather than horizontal movement or reduction. The safest way to remove debris from someone that is buried should involve carefully lifting the debris from above the victim and continually shoring the sides of the entrance hole or excavation to ensure against additional collapse.

In the case of building collapses, the magnitude of the shoring efforts and the type of equipment necessary to perform the rescue may be very different from the normal extrication. In order to facilitate vertival removal of debris, it may be essential to quickly locate and utilize various types of cranes and other types of overhead lift capabilities.

It is strongly suggested that every rescue system have previous knowledge of and training for the use of heavy construction equipment. Emergency dispatchers should have access to a list of construction companies and other businesses that could provide this type of equipment on a 24 hour a day - seven days a week basis.

\section{CONCLUSION}

Government's failure to implement reports and the sanctions recommended on collapsed buildings has been blamed for the growing rate of building collapses in the country. Since the first cases of building collapse was recorded up until the latest one in Lagos, no single individual or group has been indicted in any of them. If the government is serious and not actually playing lip service, to its transformation agenda, it must provide and ensure that the private sector also provides safe structures for people to dwell in.

\section{Recommendations}

1. The government at all levels should evolve the right policy framework for transformative urban development especially with regards to quality control at the construction stages of buildings.

2. More awareness campaigns should be carried out by the three tiers of government and their agencies on the need for compliance with the appropriate building regulations (e.g. among contractors, professionals, stakeholders and the general public) and the danger associated with their non-compliance/evasion.

3. The town planning authorities should maintain competent professionals in the relevant areas for design approval and from a long term perspective, provide the necessary training moreover; there should be regular monitoring visits to all the construction sites with a view to ensuring compliance with the approved building plans.

4. More attention should be focused towards the private or informal sector, with a view to curbing their excesses. 
5. Policy makers in the country should be less suspicious of professional advice as such advices are based on predetermined principles and deviation which may result in predictable consequence in the near future.

6. There is a need to for further awareness-raising on the dangers in patronizing incompetent people for construction activities.

7. Government should be ready to tackle the problem of building neglect in the form of legislation, enforcement, support and assistance, publicity and education.

8. Stiffer penalties (such as jail or death sentence) should be placed on owners of collapsed buildings and the project handlers in the country, especially where loss of lives is involved. It will serve as a deterrent to the practices of carefree developers in the country.

\section{References}

[1] Ayuba P., Olagunju R.E, and Akande O.K, (2007). Collapse of Buildings in Nigeria: The role of professionals and other participants in the building industry. The $1^{\text {st }}$ National Conference of the School of Environmental Technology (SET) $28^{\text {th }}$ February $-2^{\text {nd }}$ March, 2007.

[2] Baum A. and Crosby, N. (1998). Property investment appraisal, London, routledge.

[3] Chinwokwu, G. (2000). the role of professionals in averting collapse. Proceedings of a 2 day seminar of the Nigerian institute of building (Lagos state chapter) on building collapse, causes, preventing \& remedies, Lagos airport hotel, Ikeja, Lagos, $3^{\text {rd }}-4^{\text {th }}$ may.

[4] Haruna P.B. (2007). Building collapse and rescue operation: a guide towards effective performance of search and rescue operations in building collapse. The $1^{\text {st }}$ National Conference of the School of Environmental Technology (SET) $28^{\text {th }}$ February $-2^{\text {nd }}$ March, 2007.

[5] Jolaoso, B.A (accepted for publication 2004). Preventing incidences of collapse buildings in Nigeia. Journal of the Environmental technology, Federal University of Technology, Akure.

[6] Onyemachi T., Uji Z., Journal of the association of architectural educators in Nigeria $4(1)(2005) 57-63$.

[7] Olagunju R. E. O., Journal of science, technology and mathematics 5(1) (2002).

[8] Olabosipo F. I., Adedamola O. O., Journal of sustainable development 3(4) (2010) 268-277.

[9] Adebayo, S. O. (2000). Improving Building Techniques, Proceedings of a Workshop on Building Collapse: Causes, Prevention and Remedies. The Nigerian Institute of Building, Lagos State Chapter, April.

[10] Ademoroti, G. (1991). Minimizing the Collapse of Buildings in Lagos State. Proceedings of the National Seminar on effective Contract Management in Construction Industry, Nigerian Institute of Building, 22-23 August, pp. 174-187.

[11] Amusan, J. O. (1991). Strategies for Enhancing the Local Governments Roles in Minimizing the Collapse of Buildings. Proceedings of the National Conference on Effective Contract Management in the Construction Industry. Nigerian Institute of Building, 22-23August, pp. 188-200. 
[12] Badejo, E. (2009). Engineers, Others Urge Multi-Disciplinary Approach to Curb Building Collapse, The Guardian Newspaper, 13 July, pp 15-17.

[13] Chinwokwu, G. (2000). The Role of Professionals In Averting Building Collapse, Proceedings of a Seminar on Building Collapse in Nigeria. The Nigerian Institute of Building, Lagos, pp. 12-28.

[14] Dare, S. (2001). Building Design, Buildability and Site Production. Proceedings of a Workshop on Building Collapse: Causes, Prevention and Remedies, The Nigerian Institute of Building, Ondo State, 23-24October.

[15] Fadamiro J. A., Journal of Sustainable Development 3(4) (2001).

[16] Folagbade, S. O. (2001). Case Studies of Building Collapse In Nigeria. Proceedings of a Workshop on Building Collapse, Causes, Prevention and Remedies, The Nigerian Institute of Building, Ondo State Chapter, 23-24 October.

[17] Honby, A.S. (2000). Oxford Advanced learner's Dictionary. Oxford University Press, 6th Ed. p. 952.

[18] Makinde, F. A. (2007). Minimizing the Collapse of Building in Nigeria. Seminar Paper, Faculty of Environmental Studies, Osun State College of Technology, Esa-Oke, 20-21 August.

[19] Makinde, R. (1998). Building Collapse in Nigeria. A Workshop Paper on Building Collapse, Nigerian Institute of Building, Osun State Chapter, 22- 24 November.

[20] Uwakweh, B.O. (2000). Conceptual Framework for Motivating Construction workers in Developing Countries. Proceedings of the 2nd International Conference of the CIB Task Group on Construction in Developing Countries, Faculty of Engineering and Technology, University of Botswana, Botswana, 15-17 Nov., pp. 31-37.

[21] Ward, P.A (1979). Organizing and Proceedings in the Construction Industry, Plymouths MacDonald and Evans Ltd, pp. 135-137. 